\title{
Antibacterial and Resistance Modifying Activities of Nigella sativa Essential Oil and its Active Compounds Against Listeria monocytogenes
}

\author{
AHMAD MOUWAKEH ${ }^{1}$, ÁGNES TELBISZ $^{2}$, GABRIELLA SPENGLER ${ }^{3}$, \\ CSILLA MOHÁCSI-FARKAS ${ }^{1}$ and GABRIELLA KISKÓ ${ }^{1}$ \\ ${ }^{I}$ Department of Microbiology and Biotechnology, Szent Istvan University, Budapest, Hungary; \\ ${ }^{2}$ Institute of Enzymology, Hungarian Academy of Science, Budapest, Hungary; \\ ${ }^{3}$ Department of Medical Microbiology and Immunobiology, University of Szeged, Szeged, Hungary
}

\begin{abstract}
Background/Aim: N. sativa essential oil (EO) and its compounds (thymoquinone, carvacrol and p-cymene) have a broad antimicrobial spectrum. The aim of this study was to investigate the antimicrobial and resistance modifying activity of $N$. sativa EO, thymoquinone, carvacrol and p-cymene against Listeria monocytogenes. Materials and Methods: $N$. sativa EO, thymoquinone, carvacrol and p-cymene was assessed for its antimicrobial activity, modulation of antimicrobial resistance, inhibition of antimicrobial efflux and membrane integrity by broth microdilution, ethidium bromide accumulation and LIVE/DEAD BacLight ${ }^{T M}$ assays. Results: L. monocytogenes showed substantial susceptibility toward $N$. sativa EO, thymoquinone, and carvacrol. A significant reduction in MIC's of EtBr and ciprofloxacin was noticed when tested in combination with $N$. sativa EO, thymoquinone, carvacrol and reserpine. In the presence of each compound the membrane integrity was disintegrated, and the EtBr accumulation increased which was comparable to positive control reserpine. Conclusion: $N$. sativa EO might have a potential for controlling the antibiotic resistance in Listeria.
\end{abstract}

More than 200 known diseases are transmitted through food by a variety of agents that include bacteria, fungi, viruses, and parasites. Food quality and safety is becoming an important public health issue due to the increased risk of

This article is freely accessible online.

Correspondence to: Gabriella Kiskó, Ph.D., Department of Microbiology and Biotechnology, Szent István University, H-1118 Budapest, Somlói út 14-16, Hungary. Tel: +36 13057201, Fax: +36 13057340, e-mail: gabriella.kisko@etk.szie.hu

Key Words: N. sativa essential oil, Listeria monocytogenes, antimicrobial activity, resistance modifying activity. foodborne illness over the last 20 years (1) and rapid spread of multidrug resistant (MDR) bacteria. This has necessitated the discovery of new antibacterial and resistance modifying agents (2).

Bacterial multidrug efflux pumps help bacterial populations to increase their resistance and survive in the presence of antimicrobial substances. Efflux pumps (EPs) are transporter proteins found in Gram-positive and Gram-negative bacteria, which are involved in the removal of toxic substances from the interior of the cell to the external environment $(3,4)$. The main cause for MDR bacteria is the overexpression of EPs able to extrude two or more unrelated antibiotics, prior to reaching their intended targets (5). Ethidium bromide (EtBr) and other fluorescent molecules, are used as substrates extruded by EPs to demonstrate efflux activity $(6,7)$.

Listeria monocytogenes is one of the most important foodborne pathogen that has been isolated from a variety of food products (8). Two efflux pumps have been described in $L$. monocytogenes (9). The efflux pump designated as MdrL, can extrude antibiotics (macrolides and cefotaxime), heavy metals, and $\mathrm{EtBr}$ (10). Another efflux pump, termed as Lde, is associated with fluoroquinolone resistance and, in part, with resistance to acridine orange and $\mathrm{EtBr}(11)$.

The interest in plant products as alternative antimicrobial agents to control pathogenic microorganisms has been increased due to the big number of antibiotic resistant bacteria (12). A major group of plant antimicrobial compounds is represented by essential oils, which are complex mixtures of volatile secondary metabolites. They are used in the food industry because of their preservative potency and their antimicrobial effect against food-borne pathogens. Essential oils and their bioactive compounds, besides their antimicrobial effect, can increase antimicrobial activity of some antibiotics (13).

Nigella sativa essential oil contains significant amounts of phenolic compounds (i.e., $p$-cymene (p-cy), carvacrol (Car) 
in vivo $32: 737-743(2018)$

Table I. Minimal inhibitory concentration in $\mu \mathrm{g} / \mathrm{mL}$ of $N$. sativa essential oil, thymoquinone, carvacrol, and p-cymene on nine L. monocytogenes strains.

\begin{tabular}{|c|c|c|c|c|c|}
\hline L. monocytogenes strain & Source & N. sativa essential oil & Thymoquinone & Carvacrol & p-cymene \\
\hline L2 & $11 / 4.12$ t03 & 116 & 40 & 150 & $>2144$ \\
\hline L6 & $\mathrm{E} \mathrm{ST} / 10.12 .2$ & 116 & 40 & 150 & $>2144$ \\
\hline L14 & $10 / 10.12 .2$ & 116 & 40 & 150 & $>2144$ \\
\hline L7 & $\mathrm{E} \mathrm{ST} / 10.12 .3$ & 233 & 40 & 150 & $>2144$ \\
\hline L4 & E ST/10.12.1 & 233 & 40 & 150 & $>2144$ \\
\hline L1 & E $12 / 10.12 .1$ & 233 & 40 & 150 & $>2144$ \\
\hline L9 & 8/4.12.t0 & 233 & 40 & 150 & $>2144$ \\
\hline LI & NCTC, serotype $4 b$ & 466 & 40 & 150 & $>2144$ \\
\hline LA & CCM4699 & 233 & 40 & 300 & $>2144$ \\
\hline
\end{tabular}

and thymoquinone (Thq)), that might be the reason of the antimicrobial potential of Nigella sativa essential oil (14). Thymoquinone is one of its most active constituent and has different beneficial properties. Focusing on antimicrobial effects, different extracts of $N$. sativa as well as Thq, have a broad antimicrobial spectrum including Gram-negative, Gram-positive bacteria, viruses, parasites and fungi (15).

The effect of reserpine on efflux activity is often used as the benchmark to which the activities of novel efflux pump inhibitors are compared. Reserpine functions as an inhibitor of many MDR efflux pumps, although the exact mechanism of this activity has not been clarified.

This study reports the antibacterial and resistance modifying activities of $N$. sativa essential oil and its active component thymoquinone, carvacrol, and p-cymene alone and in combination with erythromycin, ciprofloxacin and EtBr against nine L. monocytogenes strains.

\section{Materials and Methods}

Cultures. Nine strains of Listeria monocytogenes listed in Table I were used for the study. Strains were from the culture collection of the Department of Microbiology and Biotechnology, Faculty of Food Science, Szent István University, Budapest, Hungary. Each strain of L. monocytogenes was individually cultured in Tryptic Soy Broth (TSB).

Chemicals. Erythromycin (Ery), ciprofloxacin (Cip) and ethidium bromide (EtBr) were obtained from Sigma Aldrich Co. (Schnelldorf, Germany). Erythromycin and ciprofloxacin were dissolved in dimethyl sulfoxide (DMSO) in order to prepare the stock solutions, which were diluted with TSB to get the appropriate concentration. EtBr was dissolved in sterile distilled water to prepare the stock solution and then diluted with TSB to get the appropriate concentration.

Essential oil and active compounds preparation. N. sativa seeds were purchased from local Turkish market and were cold pressed to produce the crude oil. The crude oil was then hydro-distilled at $100^{\circ} \mathrm{C}$ in a Clevenger apparatus to extract the essential oil (EO). The essential oil was collected, dried over anhydrous sodium sulfate and stored finally in refrigerator for further analysis.

Thymoquinone and carvacrol were purchased from Sigma Aldrich Co. and p-cymene was purchased from Alfa Aesar (Karlsruhe, Germany). The essential oil and thymoquinone were diluted using DMSO, while ethanol (EtOH) was used as diluent for carvacrol and p-cymene to prepare the stock solutions and further on diluted with TSB to get the appropriate concentration.

Antimicrobial activity. The antimicrobial activity of $N$. sativa EO, thymoquinone, carvacrol, and p-cymene were determined using the broth microdilution method (16). Briefly, the stock solution of the essential oil, thymoquinone, carvacrol or p-cymene were serially half diluted in TSB in microtiter plates, and then each $L$. monocytogenes overnight culture was added at a concentration of $10^{6} \mathrm{CFU} / \mathrm{ml}$, to the final volume of $0.1 \mathrm{ml} /$ well. After $24 \mathrm{~h}$ of incubation at $37^{\circ} \mathrm{C}, 10 \mu \mathrm{l}$ of resazurin reagent, which consisted of $10 \mathrm{M}$ resazurin sodium salt and $0.8 \mathrm{mM}$ menadione, was added to each well. Following a $2 \mathrm{~h}$ incubation at $37^{\circ} \mathrm{C}$, the fluorescence intensity was measured at $550 \mathrm{~nm}$ and $959 \mathrm{~nm}$, using a microplate reader (Victor x3, PerkinElmer, Waltham, MA, USA). The minimum inhibitory concentrations (MICs) were defined as the minimal concentration at which the fluorescence signal declined to the level of the blank. All the MICs measurements were carried out in triplicates. The diluent controls were obtained by preparing culture medium with the bacterial suspension and DMSO or EtOH with the bacterial suspension corresponding to the highest concentration present in the preparation. The negative control was obtained by preparing the culture medium only or the culture medium with the given antimicrobial. The same assay was used for the determination of the MICs for the antibiotics and EtBr.

Resistance modulation assay. Modulation of antimicrobial resistance for the antibiotics, erythromycin, ciprofloxacin, and EtBr was evaluated using the same microdilution method, except that the medium was supplemented with sub-inhibitory concentration of the essential oil and thymoquinone (1/2 MIC). The modulation factor was defined as the ratios of the MICs for the antimicrobials (Ery, Cip or EtBr) alone and for the antimicrobial agent in the presence of the EO, Thy, Carand p-cy. All the MICs measurements were carried out in triplicates. The chemical EPI, Reserpine $(20 \mu \mathrm{g} / \mathrm{ml})$ was used as positive control reference. The positive control wells 
Table II. Minimum inhibitory concentration values and modulation factors for L. monocytogenes strains.

\begin{tabular}{|c|c|c|c|c|c|c|c|c|c|}
\hline \multirow[t]{2}{*}{ Antimicrobial } & \multicolumn{9}{|c|}{ Listeria monocytogenes strain MIC $(\mu \mathrm{g} / \mathrm{ml})$} \\
\hline & $\mathrm{L} 2$ & L6 & L14 & L7 & L4 & L1 & L9 & LI & LA \\
\hline $\mathrm{EtBr}^{\mathrm{a}}$ & 128 & 64 & 128 & 128 & 128 & 128 & 128 & 128 & 64 \\
\hline $\mathrm{MF}(+\mathrm{EO})$ & 16 & 8 & 16 & 8 & 4 & 8 & 4 & 4 & 8 \\
\hline MF (+Thq) & 8 & 8 & 8 & 8 & 8 & 8 & 8 & 4 & 8 \\
\hline MF (+Car) & 16 & 16 & 8 & 8 & 16 & 16 & 8 & 4 & 2 \\
\hline MF (+p-cy) & 2 & 4 & 4 & 2 & 2 & 2 & 2 & 2 & 4 \\
\hline MF (+Res) & 8 & 8 & 8 & 8 & 8 & 8 & 8 & 8 & 8 \\
\hline Erya & 0.125 & 0.125 & 0.125 & 0.25 & 0.25 & 0.25 & 0.25 & 0.25 & 0.25 \\
\hline $\mathrm{MF}(+\mathrm{EO})$ & 2 & 2 & 2 & 2 & 4 & 2 & 2 & 4 & 2 \\
\hline MF (+Thq) & 2 & 2 & 2 & 4 & 2 & 2 & 2 & 2 & 2 \\
\hline MF (+Car) & 2 & 2 & 2 & 4 & 2 & 2 & 2 & 2 & 2 \\
\hline MF (+p-cy) & 2 & 2 & 2 & 1 & 1 & 2 & 2 & 2 & 2 \\
\hline MF (+Res) & 1 & 1 & 1 & 1 & 1 & 1 & 1 & 1 & 1 \\
\hline $\mathrm{Cip}^{\mathrm{a}}$ & 1 & 0.25 & 0.25 & 0.5 & 0.25 & 0.25 & 0.125 & 0.25 & 0.25 \\
\hline $\mathrm{MF}(+\mathrm{EO})$ & 2 & 4 & 8 & 4 & 4 & 4 & 4 & 4 & 2 \\
\hline MF (+Thq) & 4 & 4 & 2 & 4 & 2 & 4 & 4 & 4 & 2 \\
\hline MF (+Car) & 4 & 4 & 4 & 4 & 4 & 4 & 4 & 2 & 4 \\
\hline MF (+p-cy) & 2 & 2 & 4 & 4 & 2 & 2 & 2 & 2 & 2 \\
\hline MF (+Res) & 4 & 4 & 4 & 2 & 2 & 4 & 4 & 2 & 2 \\
\hline
\end{tabular}

aMIC. bMF: Modulation factors, decrease in the MIC.

were prepared with the bacterial suspension only, the bacterial suspension and $1 / 2$ MIC of the EO, Thq, Car or p-cy and with DMSO or EtOH with the bacterial suspension corresponding to the highest concentration present in the preparation.

Ethidium bromide accumulation assay. The influence of $N$. sativa $\mathrm{EO}$, Thq, Car and p-cy on EtBr accumulation in L. monocytogenes L14 was determined (17). Briefly, $150 \mu \mathrm{l}$ of overnight culture $(4 \mathrm{ml}$ inoculum, $24 \mathrm{~h}$ ) was added to $9.9 \mathrm{ml} \mathrm{TSB}$, centrifuged at $6000 \mathrm{~g}$ for $5 \mathrm{~min}$, then the cells were two times washed and resuspended in phosphate-buffered saline (PBS) $\left(\mathrm{OD}_{600}, 0.2\right)$. N. sativa $\mathrm{EO}$, Thq, Car or p-cy were added to the culture to the appropriate concentration (1/2 MIC). After $15 \mathrm{~min}$ incubation at $37{ }^{\circ} \mathrm{C}, 96.74$ $\mu l$ of the untreated culture, the treated culture containing EO, thymoquinone, carvacrol or p-cymene, were pipetted in black microtiter plates followed by the addition of $3.26 \mu \mathrm{EtBr}$ to a final concentration of $0.5 \mu \mathrm{g} / \mathrm{ml}$. The kinetics of intracellular $\mathrm{EtBr}$ accumulation were measured at $490 \mathrm{~nm}$ and $579 \mathrm{~nm}$ using a Victor x3 plate reader (PerkinElmer), at 45-sec intervals for $1 \mathrm{~h}$. Additionally reserpine $(100 \mu \mathrm{g} / \mathrm{ml})$ was used as a positive control reference in the assay. Measurements were carried out in triplicates and the means of the last 10 times points of the measurements were used in the statistical analysis.

Membrane integrity. The influence of $N$. sativa $\mathrm{EO}$, thymoquinone, carvacrol, and p-cymene on membrane integrity of $L$. monocytogenes L14 was assessed using LIVE/DEAD Baclight ${ }^{\mathrm{TM}}$ Bacterial Viability Kits (L-7012, Molecular Probes, Eugene, Oregon, OR, USA). The BacLight ${ }^{\mathrm{TM}}$ kit is composed of two nucleic acid-binding stains: SYTO 9TM and propidium iodide (PI). SYTO 9TM penetrates all bacterial membranes and stains the cells green, while PI only penetrates cells with damaged membranes, and the combination of the two stains produces red fluorescing cells (18). Briefly, exponential phase culture was washed two times and resuspended in phosphate-buffered saline (OD600, 0.2) $1 \mathrm{ml}$ of the culture was heat treated at $80^{\circ} \mathrm{C}$ for $15 \mathrm{~min}$ to obtain the dead culture, while the treated cultures with the EO, thymoquinone, carvacrol or p-cymene were incubated at $37{ }^{\circ} \mathrm{C}$ for $15 \mathrm{~min}$. A mixture of SYTO 9TM $(6 \mu \mathrm{l})$ and propidium iodide $(6 \mu \mathrm{l})$ was added to $2 \mathrm{ml}$ filtered $(0.2 \mu \mathrm{m}$ pore size filter $)$ distilled water. This dye mixture was added to $100 \mu \mathrm{l} L$. monocytogenes culture (1:1, $v / v$ ) that was untreated or treated with $N$. sativa EO, thymoquinone, carvacrol or p-cymene or heat treated. The kinetics of propidium iodide and SYTO 9 intracellular penetration were followed by measuring the relative fluorescence units (RFU) in 60-sec intervals over $1 \mathrm{~h}$, at $490 \mathrm{~nm}$ and $535 \mathrm{~nm}$ using a microplate reader (Tecan, Männedorf, Switzerland). The experiments were carried out in triplicate in black microtiter plates. The membrane disruption (\%) was calculated from the kinetic measurements of the treated relatively to the untreated cultures over the last $10 \mathrm{~min}$ of the assay.

Statistical analysis. The results were statistically analyzed using Microsoft Excel program (2016). Comparisons of the group mean values and the significances of the differences between the groups were verified by one-way ANOVA. The results were considered significant when $p \leq 0.05$.

\section{Results}

The antibacterial activity of $N$. sativa $\mathrm{EO}$ and its active compounds were determined by broth microdilution method.

As presented in Table I, the essential oil of $N$. sativa, thymoquinone and carvacrol were active against all the 


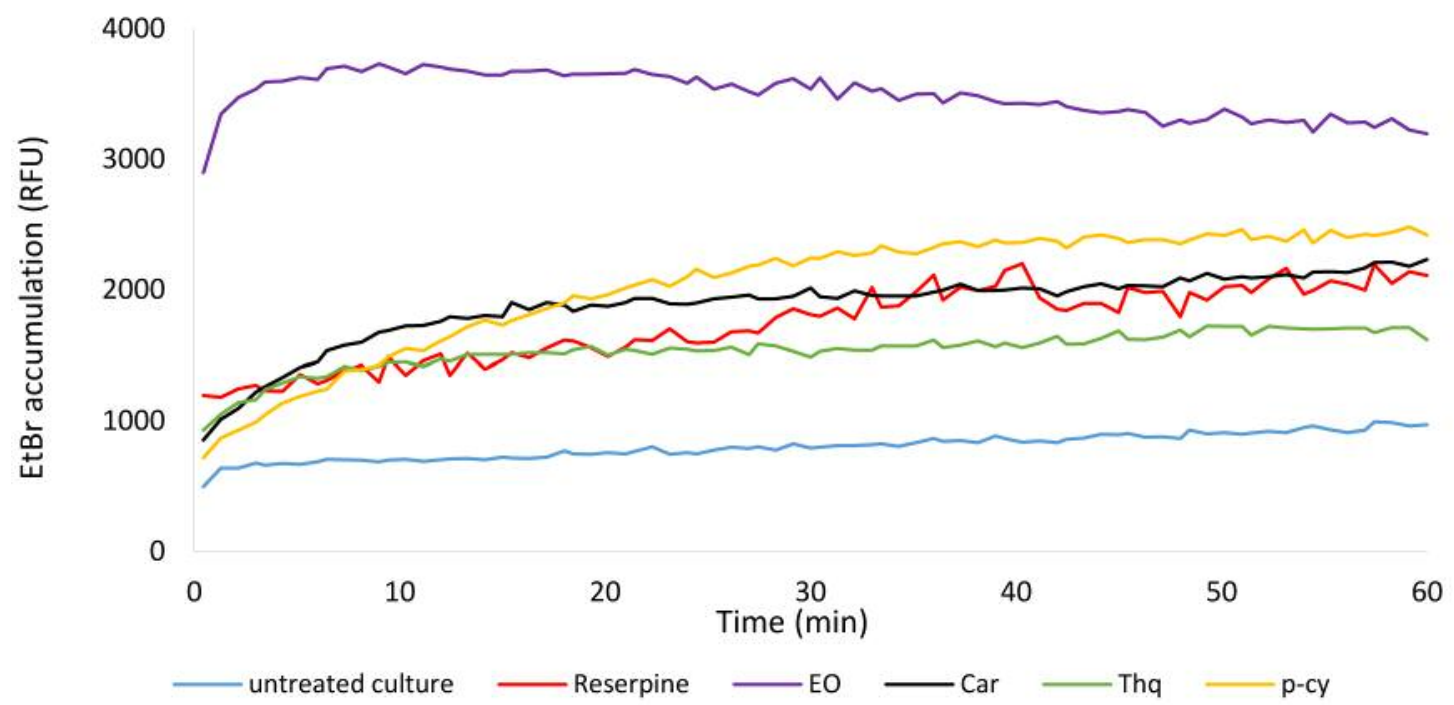

Figure 1. Ethidium bromide accumulation in L. monocytogenes L14 in the presence of sub-inhibitory concentration (1/2 MIC) of N. sativa essential oil, thymoquinone, carvacrol, and p-cymene, and reserpine as positive control. RFU: Relative fluorescence units; EtBr: ethidium bromide; EO: essential oil; Thq: thymoquinone; Car: carvacrol; $p$-cy=p-cymene.

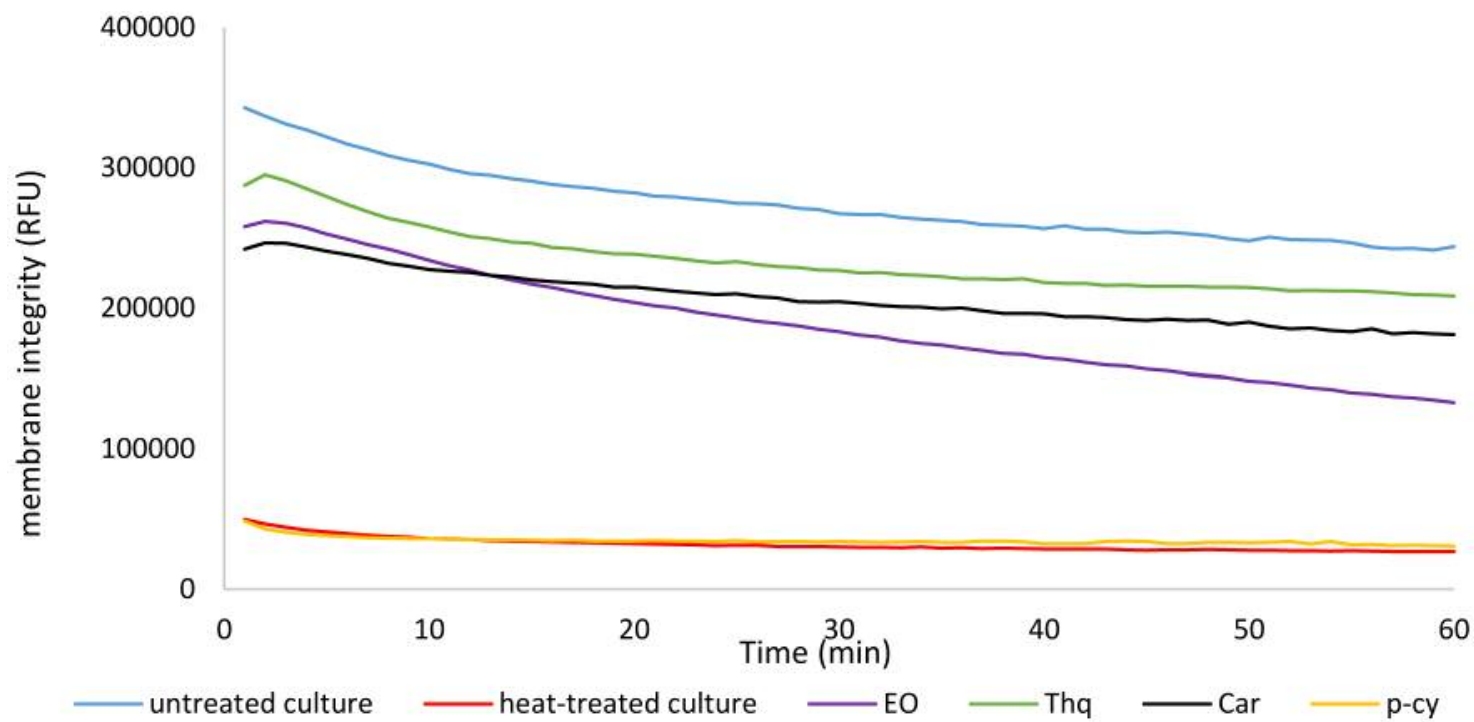

Figure 2. Influence of sub-inhibitory concentration (1/2 MIC) of $N$. sativa essential oil, thymoquinone, carvacrol and p-cymene on membrane integrity of L. monocytogenes L14. RFU: Relative fluorescent unit; EO: essential oil; Thq: thymoquinone; Car: carvacrol and p-cy: p-cymene.

tested strains of L. monocytogenes. The MIC values of $N$. sativa $\mathrm{EO}$ ranged from 116 to $466 \mu \mathrm{g} / \mathrm{ml}$, thymoquinone exhibited a significant antibacterial activity with a MIC value of $40 \mu \mathrm{g} / \mathrm{ml}$, and carvacrol exhibited the MIC of $150 \mu \mathrm{g} / \mathrm{ml}$, while p-cymene showed no inhibitory activity at the tested maximum concentration $(2,144 \mu \mathrm{g} / \mathrm{ml})$ against any strain.
As a potential modulator of antimicrobial resistance, $N$. sativa $\mathrm{EO}$, thymoquinone, carvacrol and $\mathrm{p}$-cymene were tested at $1 / 2$ MIC $(58 \mu \mathrm{g} / \mathrm{ml}, 20 \mu \mathrm{g} / \mathrm{ml}, 75 \mu \mathrm{g} / \mathrm{ml}$ and $1,072 \mu \mathrm{g} / \mathrm{ml}$, respectively) in combination with the antibiotics erythromycin, ciprofloxacin, and the antimicrobial efflux pump substrate $\mathrm{EtBr}$, on nine L. monocytogenes test strains (Table II). 
Data presented in Table II show that the supplementation of $N$. sativa $\mathrm{EO}$ (at $1 / 2 \mathrm{MIC}$ ) decreased the MICs of erythromycin from two-fold to four-fold against all L. monocytogenes strains. Additionally, a four-fold up to sixteen-fold reduction of $\mathrm{EtBr}$ was observed against L. monocytogenes strains in the presence of the EO.

Whereas, the supplementation of thymoquinone (at $1 / 2$ MIC) induced a decrease in the MIC of erythromycin from two-fold up to four-fold against seven L. monocytogenes strains and had no activity against the other three strains. Furthermore, a four-fold to eight-fold reduction of EtBr was noted against L. monocytogenes strains (Table II).

Carvacrol supplementation (at 1/2 MIC) decreased the MIC of erythromycin: two-fold reduction was observed for all the tested strains of L. monocytogenes. Additionally, a two-fold up to sixty-four-fold reduction of EtBr MIC was detected against $L$. monocytogenes strains in the presence of carvacrol (Table II).

P-cymene supplementation (at 1/2 MIC) showed two-fold reduction in the MIC of erythromycin against six $L$. monocytogenes strains and had no activity against the other four strains. P-cymene induced decrease in the $\mathrm{MIC}$ of $\mathrm{EtBr}$ from two-fold up to four-fold against all L. monocytogenes strains (Table II).

In order to elucidate the mechanism of the modulatory activity of $N$. sativa essential oil and its active compounds, thymoquinone, carvacrol, and p-cymene in L. monocytogenes L14, the potency of the compounds was evaluated to increase the accumulation of the common efflux pump substrate $\mathrm{EtBr}$ indicating efflux inhibition. The level of $\mathrm{EtBr}$ accumulation in cultures treated with half of the MIC values of $N$. sativa essential oil, thymoquinone, carvacrol, and pcymene were compared, relatively to the untreated culture, to evaluate whether it can potentiate intracellular $\mathrm{EtBr}$ accumulation. The known efflux pump inhibitor reserpine was included in the study as a positive control. The results showed significant $(p<0.0001)$ increase in the $\mathrm{EtBr}$ accumulation in the presence of $N$. sativa $\mathrm{EO}$, p-cymene, carvacrol, and thymoquinone, respectively, compared to the untreated culture of L. monocytogenes (Figure 1). N. sativa essential oil increased the EtBr accumulation significantly compared to reserpine. P-cymene and carvacrol activity were comparable to reserpine, while thymoquinone activity was lower than reserpine.

Again, 1/2 MIC of N. sativa EO, Thq, Car, and p-cymene were tested for their influence on membrane integrity in $L$. monocytogenes L14, to determine whether membrane permeability is the main mechanism of its modulation of antimicrobial resistance. The membrane integrity of cultures treated with 1/2 MIC of $N$. sativa essential oil, thymoquinone, carvacrol or p-cymene decreased by $43 \%, 14 \%, 25 \%$ and $87 \%$ respectively (Figure 2). Hence, at this half MIC concentration of the essential oil and its active compounds, the disruption of the membranes is likely to have contributed to their antimicrobial resistance modifying effect. Cultures incubated at $80^{\circ} \mathrm{C}$ for $15 \mathrm{~min}$ were used as positive controls for disrupted membranes, and these showed $89 \%$ decreased membrane integrity, compared to the untreated control cultures. These differences were calculated based on the kinetics measurements over the last $10 \mathrm{~min}$ of the $1 \mathrm{~h}$ assay, and they were statistically significant $(p<0.00001)$. The disruptive impact of p-cymene on membrane integrity is not surprising, as it is known that monoterpenes can cause alteration in membrane permeability (19).

\section{Discussion}

In general, antimicrobial resistance alters the activity of antibiotics through one of the following mechanisms: antimicrobial target modification (decreasing drug affinity), a decrease in drug absorption, activation of efflux mechanisms to expel the toxic molecules (overexpression of efflux pumps) or global changes in important metabolic pathways through the modulation of regulation networks (20). The search of new efflux pump inhibitors is necessary to combat the emergence of antibacterial resistant strains (21).

Data presented in Table I show that all tested strains were sensitive to $N$. sativa essential oil, thymoquinone and carvacrol, while p-cymene did not show any activity against the tested L. monocytogenes strains. These results support previous studies which reported an effective antibacterial activity of $N$. sativa essential oil (22), thymoquinone (23) and carvacrol (24) against Gram-positive bacteria. DMSO and $\mathrm{EtOH}$ as diluents did not show any inhibitory effect on the growth of the strains.

Efflux is an important mechanism of resistance in $L$. monocytogenes (10). The efflux pumps are proteins of bacterial membranes which extrude and limit the intracellular accumulation of antibiotics and other antimicrobial agents (5). It was previously identified that the increased resistance to ciprofloxacin (a fluoroquinolone) confers also increased resistance to $\mathrm{EtBr}$, and an increased expression of the Lde transporter (11). Our data showed the potential of $N$. sativa essential oil, thymoquinone, carvacrol, and p-cymene to decrease the MIC of ciprofloxacin at least two-fold and up to eight-fold.

Additionally, in a previous study, increased susceptibility to macrolides resulted in the inactivation of the MdrL in $L$. monocytogenes (10). The MIC of erythromycin (a macrolide) decreased in the presence of $N$. sativa essential oil, thymoquinone, carvacrol, and p-cymene at least two-fold up to four-fold, while reserpine had no activity with erythromycin.

EtBr is a substrate of many MDR pumps and causes fluorescence when bound to DNA (25). Multidrug efflux pumps are known to cause resistance to this agent, thus the 
effects of inhibition of efflux can be assessed fluorometrically $(26,27)$. As found previously, the MICs of EtBr were also lower in combination with $N$. sativa essential oil, thymoquinone, carvacrol, and p-cymene (Table II).

Nigella sativa essential oil, thymoquinone, carvacrol, and pcymene induced the increase of EtBr accumulation in the treated strain and was comparable to the chemical EPI, reserpine. The inhibition of EtBr via a number of efflux pumps has been already reported for L. monocytogenes $(10,11)$.

Since plant essential oils and their components are hydrophobic in nature, their primary target is the bacterial membrane to make it more permeable, which could permit an increased uptake of the antimicrobial agent by the bacterial cell (28).

These observations presume the modulating activity of $N$. sativa essential oil and thymoquinone through efflux pumps inhibition leading to increased accumulation of antibiotics in the cells enhancing their effects at lower doses. So, the inhibition of EtBr efflux supports the hypothesis of antibacterial activity of $N$. sativa essential oil and its active components thymoquinone, carvacrol, and p-cymene through pump efflux inhibition.

Therefore, the results presented in this study suggest that the essential oil of $N$. sativa and its major components thymoquinone and carvacrol act as putative efflux pump inhibitors in Listeria, modulating the bacterial resistance to antibiotics. To the best of our knowledge, this is the first report on resistance modifying activity of $N$. sativa essential oil against $L$. monocytogenes.

In conclusion, Nigella sativa essential oil and its active compounds, thymoquinone and carvacrol are confirmed as efficient modulators of antimicrobial resistance in $L$. monocytogenes, with at least two different mechanisms that contribute synergistically to their activity. Half MIC of $N$. sativa essential oil, thymoquinone, and carvacrol modulates antibiotic resistance in L. monocytogenes against various antimicrobials, showing increased $\mathrm{EtBr}$ accumulation. Additionally, by targeting the membrane, they caused increased permeability, thereby promoting the influx of antimicrobials. P-cymene had no antimicrobial activity; however, it increased the membrane permeability. Due to the modulation of the antimicrobial resistance in $L$. monocytogenes, $N$. sativa essential oil, thymoquinone, and carvacrol have the potential to be promising modifiers of antimicrobial resistance in L. monocytogenes.

\section{Acknowledgements}

The Project was supported by the European Union and co-financed by the European Social Fund (grant agreement no. EFOP-3.6.3VEKOP-16-2017-00005). Gabriella Spengler was supported by the János Bolyai Research Scholarship of the Hungarian Academy of Sciences.

\section{References}

1 Olivier SP, Jayarao BM and Almeida RA: Foodborne pathogens in milk and the dairy farm environment: food safety and public health implications. Foodborne Pathog Dis 2: 115-129, 2005.

2 Sweeney LC, Dave J, Chambers PA and Heritage J: Antibiotic resistance in general dental practice - a cause for concern? J Antimicrob Chemother 53: 567-576, 2004.

3 Van Bambeke F, Pages J-M and Lee VJ: Inhibitors of bacterial efflux pumps as adjuvants in antibiotic treatments and diagnostic tools for detection of resistance by efflux. Recent Pat Anti-infect Drug Discov 1: 157-175, 2006.

4 Kristiansen JE, Thomsen VF, Martins A, Viveiros M and Amaral L: Non-antibiotics reverse resistance of bacteria to antibiotics. In Vivo 24: 751-754, 2010.

5 Piddock LJ V: Clinically relevant chromosomally encoded multidrug resistance efflux pumps in bacteria. Clin Microbiol Rev 19: 382-402, 2006.

6 Kern W V, Steinke P, Schumacher A, Schuster S, von Baum H and Bohnert JA: Effect of 1-(1-naphthylmethyl)-piperazine, a novel putative efflux pump inhibitor, on antimicrobial drug susceptibility in clinical isolates of Escherichia coli. J Antimicrob Chemother 57: 339-343, 2006.

7 Raherison S, Gonzalez P, Renaudin H, Charron A, Bébéar C and Bébéar CM: Evidence of active efflux in resistance to ciprofloxacin and to ethidium bromide by Mycoplasma hominis. Antimicrob Agents Chemother 46: 672-679, 2002.

8 Jemmi T and Stephan R: Listeria monocytogenes: food-borne pathogenesis and virulence factors. Rev Sci Tech 25: 571-580, 2006.

9 Romanova NA, Wolffs PFG, Brovko LY and Griffiths MW: Role of efflux pumps in adaptation and resistance of Listeria monocytogenes to benzalkonium chloride. Appl Environ Microbiol 72: 3498-3503, 2006.

10 Mata MT, Baquero F and Pérez-Díaz JC: A multidrug efflux transporter in Listeria monocytogenes. FEMS Microbiol Lett 187: 185-188, 2000

11 Godreuil S, Galimand M, Gerbaud G, Jacquet C and Courvalin P: Efflux pump Lde is associated with fluoroquinolone resistance in Listeria monocytogenes. Antimicrob Agents Chemother 47: 704-708, 2003.

12 Hemaiswarya S, Kruthiventi AK and Doble M: Synergism between natural products and antibiotics against infectious diseases. Phytomedicine 15: 639-652, 2008.

13 Langeveld WT, Veldhuizen EJA and Burt SA: Synergy between essential oil components and antibiotics: a review. Crit Rev Microbiol 40: 76-94, 2014.

14 Hassanien MFR, Assiri AMA, Alzohairy AM and Oraby HF: Health-promoting value and food applications of black cumin essential oil: an overview. J Food Sci Technol 52: 6136-6142, 2015.

15 Forouzanfar F, Fazly Bazzaz BS and Hosseinzadeh H: Black cumin (Nigella sativa) and its constituent (thymoquinone): a review on antimicrobial effects. Iran J Basic Med Sci 17: 929938, 2014.

16 Kovač J, Katarina Š, Wu Z, Klančnik A, Bucar F, Zhang Q and Možina SS: Antibiotic resistance modulation and modes of action of (-) - $\alpha$-pinene in Campylobacter jejuni. PLoS One 10: $1-14,2015$ 
17 Kovač J, Gavaric N, Bucar F and Možina SS: Antimicrobial and resistance modulatory activity of Alpinia katsumadai seed phenolic extract, essential oil and post-distillation extract. Food Technol Biotechnol 52: 248-254, 2014.

18 Simoes M, Rocha S, Coimbra M and Vieira M: Enhancement of Escherichia coli and Staphylococcus aureus antibiotic susceptibility using sesquiterpenoids. Med Chem 4: 616-623, 2008.

19 Trombetta D, Castelli F, Sarpietro MG, Venuti V, Cristani M, Daniele C, Saija A, Mazzanti G and Bisignano G: Mechanisms of antibacterial action of three monoterpenes. J Antimicrob Agents Chemother 49: 2474-2478, 2005.

20 Munita JM and Arias CA: Mechanisms of Antibiotic Resistance. Microbiol Spectr 23: 1-24, 2016.

21 Stavri M, Piddock LJ V and Gibbons S: Bacterial efflux pump inhibitors from natural sources. J Antimicrob Chemother 59: 1247-1260, 2007.

22 Piras A, Rosa A, Marongiu B, Porcedda S, Falconieri D, Dessì MA, Ozcelik B and Koca U: Chemical composition and in vitro bioactivity of the volatile and fixed oils of Nigella sativa L. extracted by supercritical carbon dioxide. Ind Crops Prod 46: 317-323, 2013.

23 Kouidhi B, Zmantar T, Jrah H, Souiden Y, Chaieb K, Mahdouani $\mathrm{K}$ and Bakhrouf $\mathrm{A}$ : Antibacterial and resistance-modifying activities of thymoquinone against oral pathogens. Ann Clin Microbiol Antimicrob 10: 29, 2011.
24 Magi G, Marini E and Facinelli B: Antimicrobial activity of essential oils and carvacrol, and synergy of carvacrol and erythromycin, against clinical, erythromycin-resistant group A Streptococci. Front Microbiol 6: 1-7, 2015.

25 Martins M, Viveiros M, Couto I, Costa SS, Pacheco T, Fanning S, Pages JM and Amaral L: Identification of efflux pumpmediated multidrug-resistant bacteria by the ethidium bromideagar cartwheel method. In Vivo 25: 171-178, 2011.

26 Ogawa W, Li D-W, Yu P, Begum A, Mizushima T, Kuroda T and Tsuchiya T: Multidrug resistance in Klebsiella pneumoniae MGH78578 and cloning of genes responsible for the resistance. Biol Pharm Bull 28: 1505-1508, 2005.

27 Borges-Walmsley MI, McKeegan KS and Walmsley AR: Structure and function of efflux pumps that confer resistance to drugs. Biochem J 376: 313-338, 2003.

28 Carson CF, Carson CF, Mee BJ, Mee BJ, Riley TV and Riley TV: Mechanism of action of Melaleuca alternifolia (Tea Tree) oil on Staphylococcus aureus determined by time-kill, lysis, leakage, and salt tolerance assay and electron microscopy. Antimicrob Agents Chemother 46: 1914-1920, 2002.

Received February 8, 2018 Revised April 20, 2018 Accepted April 23, 2018 